\title{
Dynamic Displacements of the RHIC Dipole Cold Mass with Injection Molded Composite Posts During Quench Conditions
}

\author{
J. Sondericker and L. J. Wolf
}

February 1991

\section{DISCLAIMER}

\begin{abstract}
This report was prepared as an account of work sponsored by an agency of the United States Government. Neither the United States Guvernment nor any agency thereof, nor any of their employees, makes any warranty, express or implied, or assumes any legal liability or responsibility for the accuracy, completeness, or usefulness of any information, apparatus, product, or process disclosed, or represents that its use would not infringe privately owned rights. Reference herein to any specific commercial product, process, or service by trade name, trademark, manufacturer, or otherwise does not recessarily constitute or imply its endorsement, recommendation, or favoring by the United States Government or any agency thereof. The views and opinions of authors expressed herein do not necessarily state or reflect those of the United States Government or any agency thereof.
\end{abstract}

\section{R H J C P R O J E C T}

\author{
Brookhaven National Laboratory \\ Associated Universities, Inc. \\ Upton, NY 11973
}

Under Contract No. DE-AC02-76CH-00016 with the UNITED STATES DEPARTMENT OF ENERGY 


\title{
DYNAMIC DISPLACEMENTS \\ OF THE RHIC DIPOLE COLD MASS \\ WITH INJECTION MOLDED COMPOSITE POSTS \\ DURING QUENCH CONDITIONS
}

\author{
Jack Sondericker \\ Brookhaven National Laboratory \\ and \\ Lawrence J. Wolf \\ Superconducting Super Collider Laboratory
}

$1 / 13 / 91$

Conclusions

1. During quench, the end of the cold mass of the RHIC dipole magnet can be expected to vibrate with a maximum displacement of 8.4 thousands of an inch in the direction of the ion-beam axis.

2. The dynamic displacement at the middle is only slightly less (8.2 thousands of an inch) than at the end of the cold mass.

3. Due to the curvature of the cold mass, oscillations are also produced in the lateral and vertical directions. But, at the end of the cold mass these are only about 25 and $5 \%$, respectively, of the axial travel.

4. A good value for the lateral stiffness of a center post made of Ultem 2100 when cooled to magnet operating temperatures is about 55,000 pounds per inch, versus the previously published 45,000 pounds per inch 1 at room temperature.

1 Wolf, L.J.. Shear Tests of Injection Molded Composite Posts Simulating Hydrostatic End Loads on a Magnet Cold Mass; Brookhaven National Laboratory; Technote AD/RHIC-79; August, 1990 
5. The post stiffness at magnet operating temperatures is relatively constant over the full load range.

6. The effective pressure area of the helium containment bellows having a outside convolution diameter of 7.63 inches is 36.7 square inches.

7. Based on the above effective pressure area, the maximum amplitude of the force transient due to quench pressure differentials across the length of a cold mass is about 300 pounds.

8. The pressure differential goes through an initial cycle with a period of about .28 seconds. This is about half that of the lowest natural frequency of the cold mass. Therefore, the quench pressures do little to excite resonances in the cold mass structure.

9. While the value of damping is at this time unknown, it makes little difference in the axial and lateral excursions of the RHIC cold mass undergoing a quench.

10. The dynamic axial displacement is about two times what the static displacement of the injection molded post would be when subjected to a static pressure differential of the same value.

\section{Background}

The injection molded composite (IMC) posts are intended to replace not only the shrink-fitted fiberglass reentrant posts, but also the diagonal straps. Since the purpose of diagonal straps are to resist the end load due to pressure upset conditions, the IMC posts must be included in a structural system which will carry the end-load. The structural concept upon which the design is based is one in which the lateral bending resistance of the post becomes paramount, and the cold mass cradle becomes a part of the bending moment transfer mechanism. ${ }^{2}$

While the strength of the IMC posts have been proven in a testing program, ${ }^{3}$ there is yet some concern about their stiffness. This concern centers upon the fact that, without straps or tie bars the entire quench load must essentially be born by the single center post. Furthermore it has been asserted that since the load is dynamic rather than a static, it could excite harmonic excursions of the cold mass, due to the flexibility of the posts, which could

\footnotetext{
2 Wolf, L.J.. Structural Concept for Support of the RHIC Cold Mass Using Injection Molded Composite Posts; Brookhaven National Laboratory; Technote AD/RHIC-65; February, 1990 3 Op. Cit., Reference 1
} 
cause interference with, or damage to, other magnet components.

The new design of the RHIC dipole magnets incorporate helium containment bellows having a convolution diameter of only 7.63 inches. The present bellows are 12.80 inches in diameter. The smaller bellows present a substantially reduced pressure area which can be expected to reduce proportionately the end force on the cold mass during a quench. But, the objection was raised that the smaller bellows would present greater obstruction to the helium flow during a quench thereby producing higher pressure differentials.

This analysis was undertaken to address these assertions by predicting the dynamic displacements of the cold mass using the latest test data on the stiffness of the IMC posts, pressure-time histories acquired from the recent full cell tests of RHIC magnets, and the dimensions of the new expansion joints. The analysis treated the cold mass as an elastic body having a saggittal curvature. The technique of normal mode expansion of a lumped-parameter system was used to obtain the results and conclusions reported herein.

\section{Stiffness and Strength of the IMC Posts}

The cryostat design specification for the end load on the dipole magnet cold mass is 12500 pounds. Single IMC posts made of Ultem 2100 have met or exceeded this requirement when tested in shear in the fixed-fixed end condition as replicates the center post supporting the cold mass. The posts have recently been tested in the shear fixture shown in Figure 1 with the inboard "hat sections" on each side cooled with liquid nitrogen. Since two posts are used in this test fixture, a 25,000 pound shear load along with a 3,000 pound axial load was used to qualify the posts against the specified end load coupled with the anticipated dead weight of the cold mass. Coupon tests have shown that, between the temperatures of liquid helium and liquid nitrogen, there are only small changes in the structural properties of this material. Therefore, the cooling of the inboard hat sections with nitrogen, while insulating the walls of the outboard hat sections, can be expected to give a fairly good representation of the stiffness under the conditions which the posts must actually operate.

4 Wolf, L.J.; Sondericker, J.H.; and DiVito, W.A; Elastic Moduli of Uttem and Noryl at Cryogenic Temperatures Using Vibrating Beam Specimens, Brookhaven National Laboratory; Technote AD/RHIC/RD-21, June, 1990 
$3000 \#$

Hydraulic cylinder force

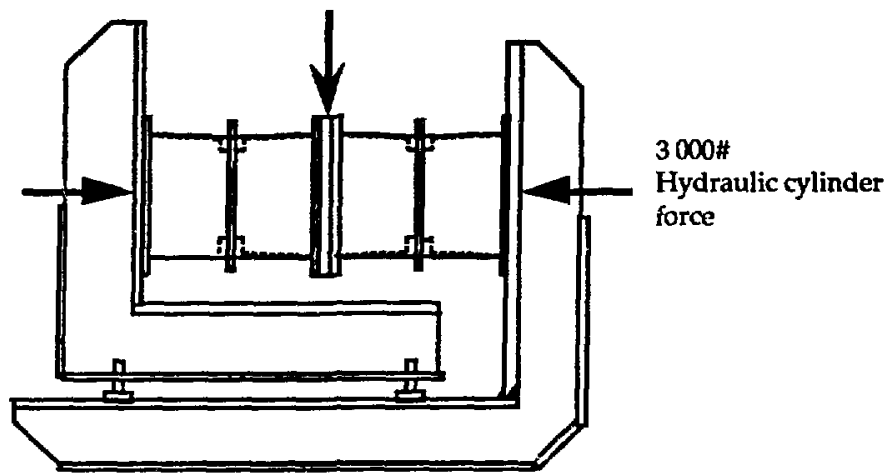

Figure 1

Two posts in the shear testing fixture.

The test results are given in Figure 2. First, the posts were tested to the 25,000 pound $(12,500$ each) specification load while cold . Deflections were measured in order to gain information about the actual stiffness of the posts. Then, the test was repeated at room temperature and was continued to failure. The warm posts in this test exceeded the strengths reported in Reference 1 . This is due to improvements in the mold and casting procedures since the last family of tests. The cold posts exhibited a stiffness of 55,000 pounds per inch, versus 40,500 warm. Equally important, the nonlinearity of the forcedeflection curve which appears at higher loads when the posts are tested warm, is greatly reduced when cold.

\section{End Force Transinitted by Bellows}

To arrive at a realistic estimate of the force due to a pressure differential, a convolution of the bellows was treated as a pair of toroidal quadrants connected by an annular plate. These plate and shell pieces were modeled, as shown in the Figure A1 of the Appendix, with 30 axisymmetric shell elements. The MSC/PALS finite element software5, which is essentially a NASTRAN program for the personal computer, was used.

5 MSC/PALS, Advanced Stress and Vibration Analysis Reference Manual, Apple Macintosh Version, The MacNeal-Schwendler Corporation, Los Angeles, 1989 
The finite element analysis of the bellows convolution produced the result in Figure 3. The cold mass would have to provide the reactions shown at the upper face of the convolution. The cold mass, in the case of differential pressure, must provide $2.85 \#$ of wall force force for each psi. of pressure plus the pressure acting over the 6.63 inch diameter circular area in order to equilibriate the bellows. This produces an effective area of 37 square inches, or an effective diameter of 6.83 inches. (The reducing effect of the beam tube bellows was neglected. )

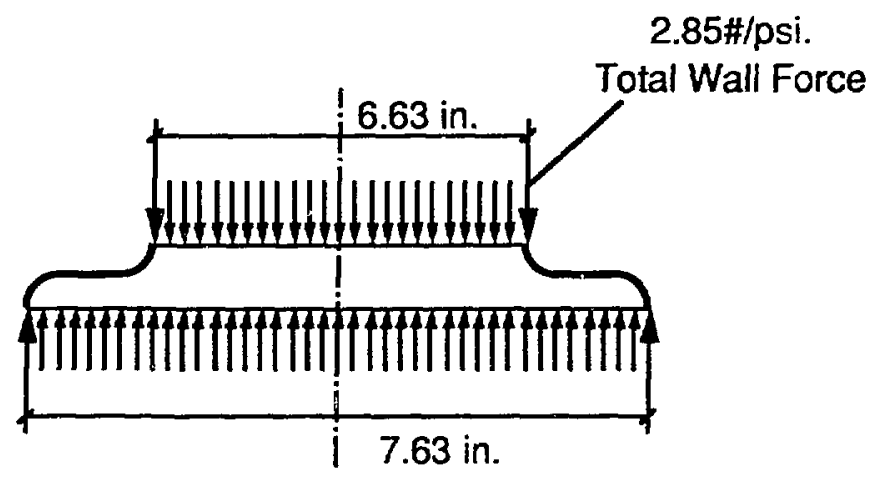

Figure 3

Forces due to pressure on the bellows convolutions.

\section{Pressure Transients Due to Quench}

The pressure-time histories during a quench were obtained from transducers installed at the locations shown during a full cell test. The maximum differential pressure occurred across Dipole 008 at a quench current of 6600 amps. The differential pressures were reported by J. G. Cottingham" to be "an extreme test of the quench generated differential pressure across a dipole in RHIC."

- Cottingham, J.G., Dipole Differential Pressures During Quench; Brookhaven National Laboratory: Memo to J. Sondericker; December 14, 1990 


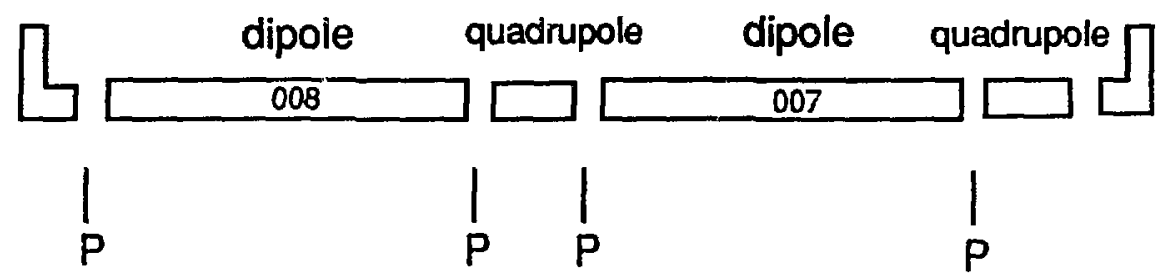

Figure 4

Pressure measurement points during full-cell test.

The maximum dynamic pressure differential was 8 psi. above the 1 psi. steady-state-flow value. For this study the initial steady-state value was taken as zero.

While it can be argued that these differential pressure measurements were obtained using the larger bellows, and that the new smaller bellows will offer a greater impediment to the equalization of quench pressures throughout the full-cell system, that effect should be slight. The ratio of the length over the cross sectional area of the helium flow channel through the dipole cold mass is about 120. The ratio through the new bellows is only .90. Therefore, most of the pressure differential will continue to oscur over the length of the cold mass rather than across the bellows. The pressure differentials reported by Cottingham can be expected to be representative of the same test using the smaller bellows.

\section{Dynamic Response of the RHIC Cold Mass Due to the Quench Pressure}

The cold mass was modeled with 13 lumped masses supported on three elastic posts as shown in Figure A2 of the Appendix. The saggitta of 1.877 inches on the 380 inch long cold mass was included in positioning these masses. The posts are spaced 141.5 inches apart in a straight line which is offset 1.195 inches from the apex of the saggittal arc. The post connection nodes are offset to the true positions of their intersections in space, even though their elastic lengths are those of the hat sections. The elasticity between the lumped masses is that provided by the stainless steel helium containment shell. The core pieces, coils and beam tube were assumed to contribute only to the mass and not to stiffness.

An effective modulus of elasticity was computed so that the center post has a shear stiffness of 55,000 pounds/inch. The side posts have the same vertical and lateral stiffness as the center post, but they have a release in the direction 
of the ion-beam axis. A modal damping of .003 of critical was used as was obtained from a vibrating beam of Ultem 2100 at the temperature of liquid helium. ${ }^{7}$ This is a material damping and it assumes, accurately as it turns out, that most of the flexing during vibration is in the posts.

The differential pressure record for the 2.5 seconds after the quench was digitized at .02 second intervals and multiplied by the effective area of 37 square inches. The resulting axial force transient is included in Figure A3 of the Appendix. The maximum force is 323 pounds and occurs after an initial cycle having a period of about 28 seconds. A similar pulse shape, though with a slightly lower amplitude, was produced across the Dipole 008 during another quench which initiated in a different part of the full-cell. This pulse shape seems to be characteristic.

The lumped parameter system of Figure A2 was subjected to the force transient of Figure A3, yielding the displacement responses given in Figures A4 and A5 of the Appendix. The maximum and minimum travels from these figures are:

$\begin{array}{ccc} & \begin{array}{c}\text { Maximum } \\ \text { Axial } \\ \text { Displacement } \\ \text { (Inches) }\end{array} & \begin{array}{c}\text { Minimum } \\ \text { Axial } \\ \text { Displacement } \\ \text { (Inches) }\end{array} \\ \text { End of Cold Mass } & 8.40 \mathrm{E}-3 & -3.47 \mathrm{E}-3 \\ \text { Center of Cold Mass } & 8.28 \mathrm{E}-3 & -3.42 \mathrm{E}-3\end{array}$

Figure A5 shows that there is substantial coupling between the lateral vibration modes and the axial modes due to the saggittal curvature of the cold mass. The end of the cold mass can be expected to have a transient displacement of about two thousands of an inch in the lateral direction, or $25 \%$ of the axial displacement. There is a lessor coupling with the vertical modes. The dynamic vertical displacement is less than $5 \%$ of the axial.

The above dynamic displacements are the result of the excitation of the first five modes of vibrations. Their natural frequencies are 6.712, 8.146, 12.15, 12.62 , and $14.36 \mathrm{~Hz}$. The mode shapes corresponding to these frequencies are given in Figure A6 of the Appendix. The analysis was done using a normal mode expansion performed by the MSC/PALS software ${ }^{8}$ on a Macintosh computer.

The Effects of Damping

7 Op. Cit., Reference 4

Op. Cit., Reference 5 


\section{The Effects of Damping}

Damping in the actual magnets will come from three sources; material damping, coulomb damping, and viscous damping. Only the material damping from the Ultem has been accounted for here. The microscopic sliding of the core laminates against one another as the cold mass flexes laterally can be expected to contribute coulomb damping. The axial motion of the cold mass necessarily requires that some liquid helium be transferred from one bellows chamber, through the cold mass, to the bellows chamber at the other end. Even though liquid helium has a very low viscosity, it is hard to conceive of a better physical configuration for a viscous damper.

However, the relative frequency content of the pressure transient and of the elastic response make any discussion of damping superlative to the question at hand. Because the excitation frequency is significantly lower than the natural frequencies of the system, additional damping only serves to make the oscillations die out in fewer cycles. It does not materially change the peak amplitude of the oscillations.

This was demonstrated by submitting a modal damping of $10 \%$ of critical damping to the analysis. Increasing the damping by 30 times reduces the maximum value of displacement of the end of the cold mass by only $5 \%$.

Due to the relative frequencies of the transient and the structural system, the maximum displacement of the systrm is about twice what the displacement would be due to a static pressure of the same level. Damping effects it only a little. This means that the quench transients fall into the structural category of "sudden loads" where a factor of two is conventionally used on the applied loads. This is in contrast to "cyclic loads" near resonance, or "shock loads" having frequency content much higher than the resonances. In those latter cases the accuracy of the damping values are much more important in assessing the displacements and the dynamic stresses.

APPENDICES

Figure A1 Pressure on Bellows Convolution

Figure A2 Coldmass Lumped Parameters

Figure A3 Force Due to Quench

Figure A4 Mid-Coldmass Travel

Figure A5 Coldmass End Travel

Figure A6 Natural Modes of Vibration 
Fig. A1 Pressure on Bellows Convolution
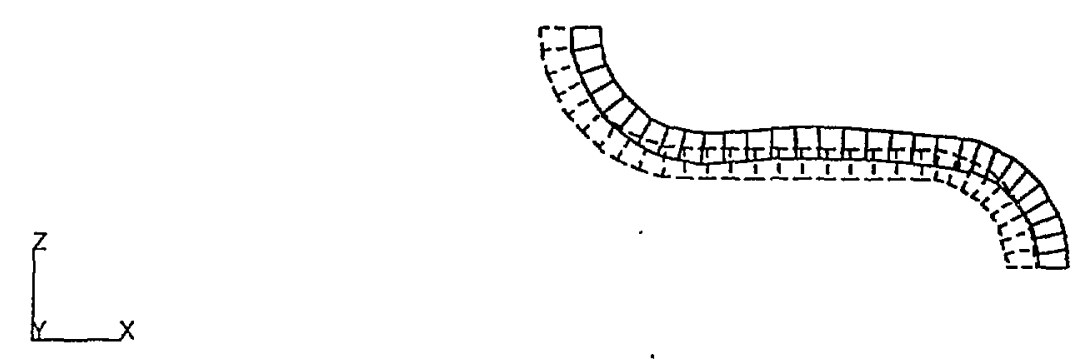

Fig. A2 Coldmass Lumped Parameters

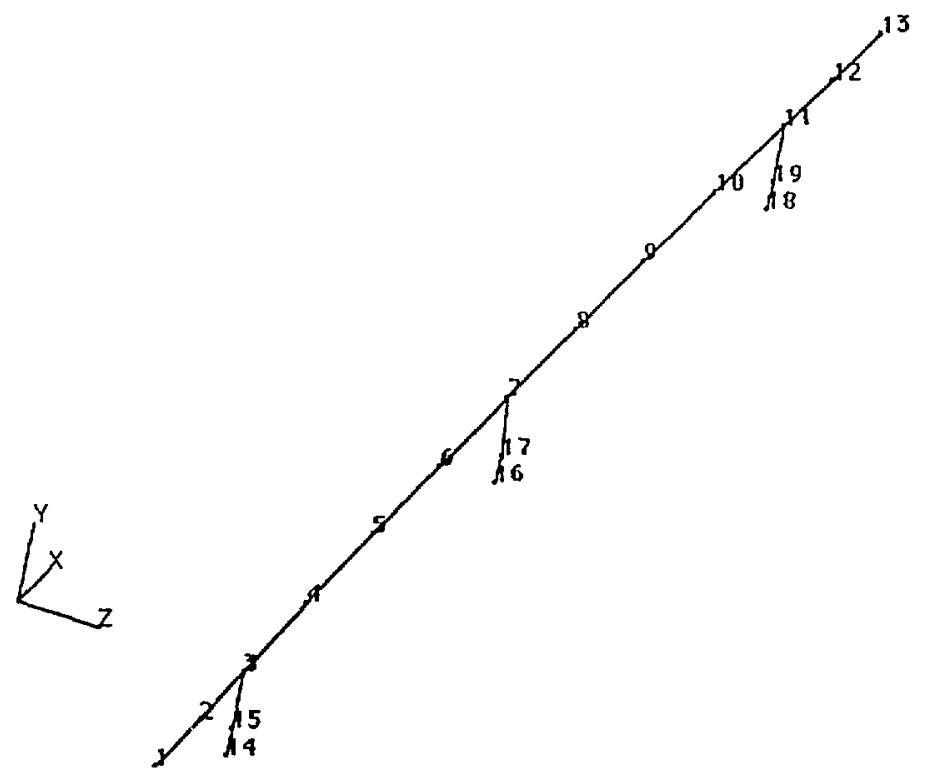




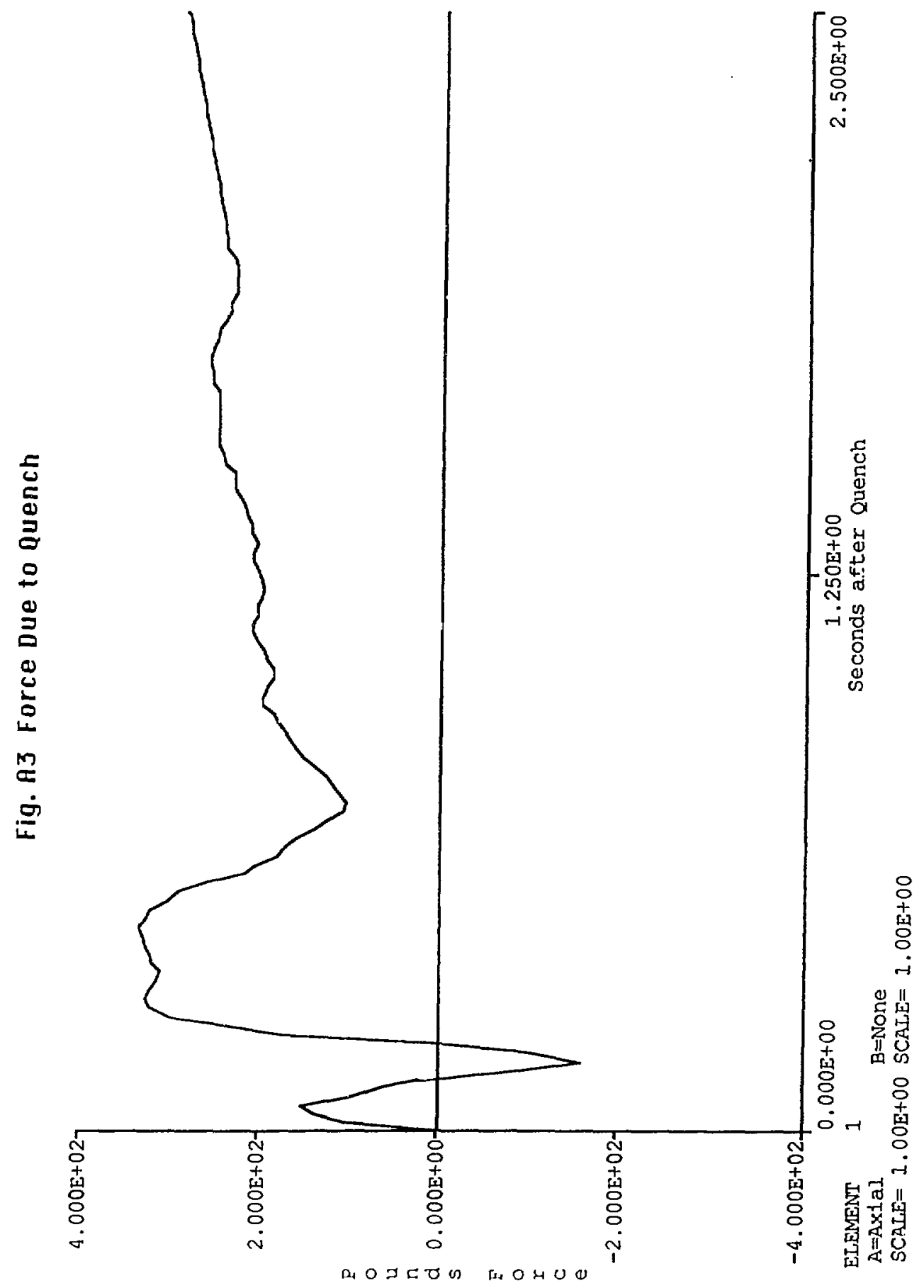




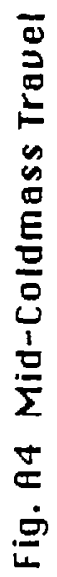
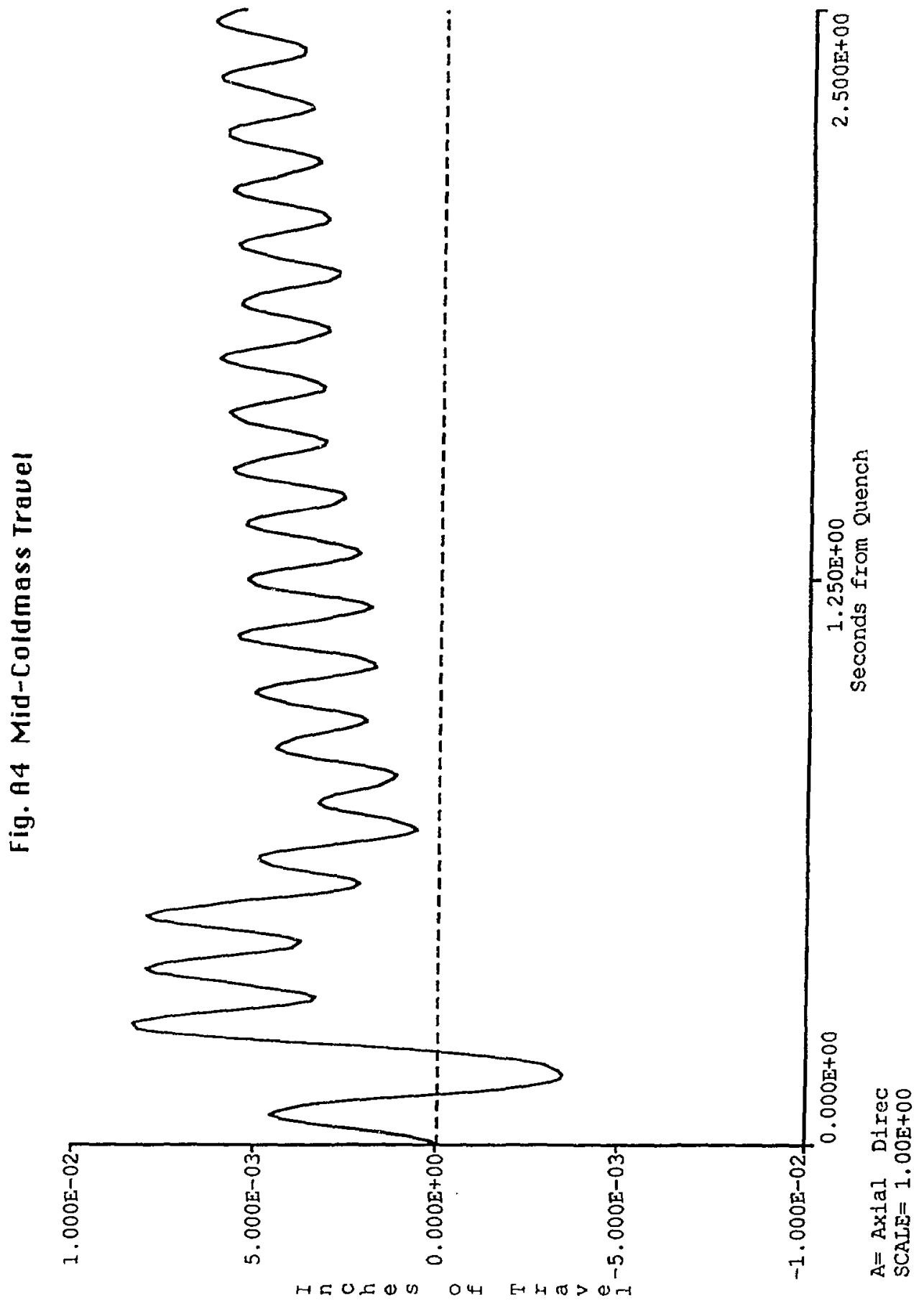
Fig. A5 Coldmass End Travel

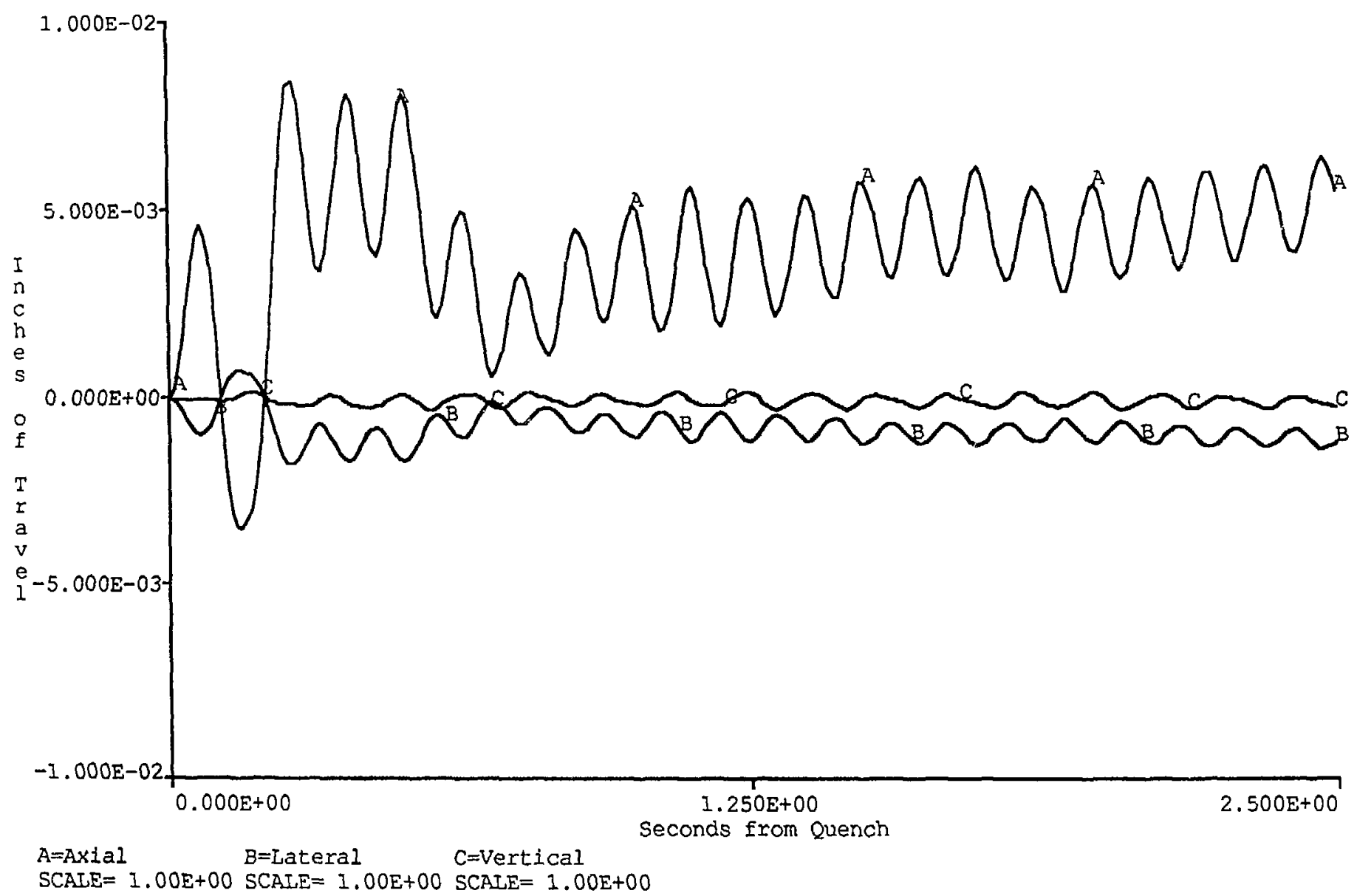




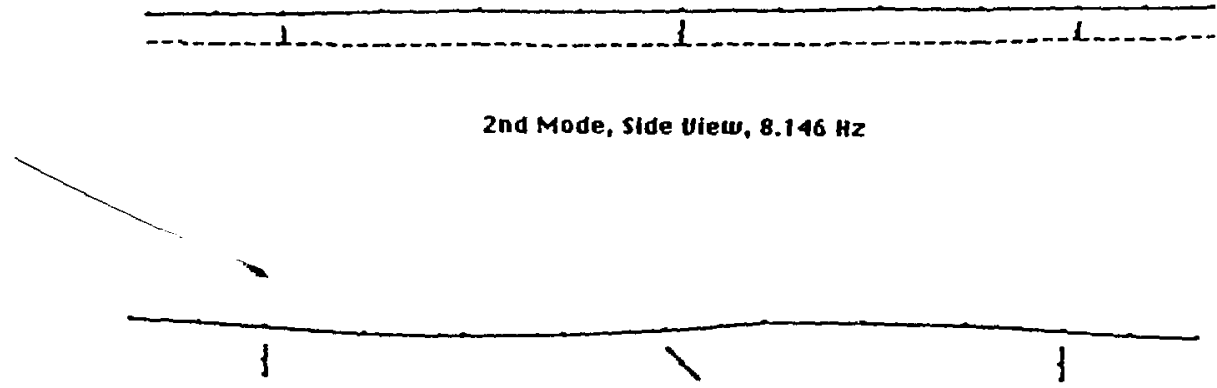

3rd Mode, Top Uiew, $12.15 \mathrm{~Hz}$

4th Mode, Top View, $12.62 \mathrm{kz}$

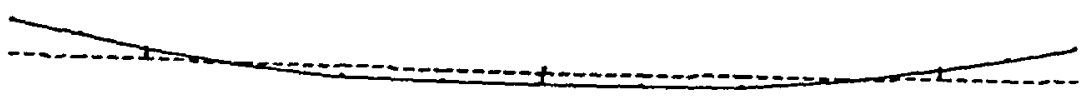

Sth Mode, Top UJew, $14.36 \mathrm{~Hz}$

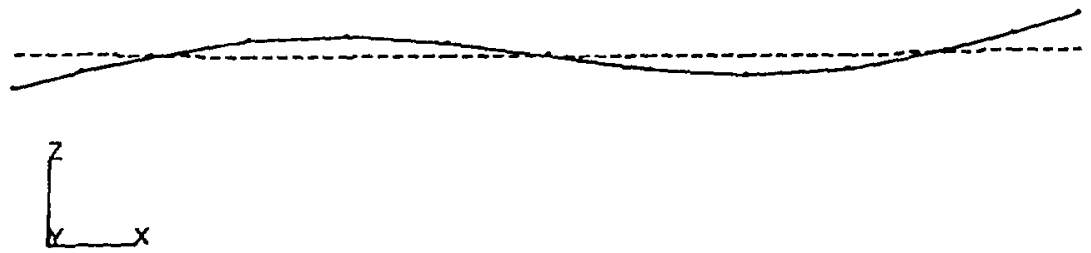

